\title{
Using Empirical Data to Estimate Potential Functions in Commodity Markets: Some Initial Results
}

\author{
C. Shen ${ }^{1} \cdot$ E. Haven ${ }^{1}$
}

Received: 22 December 2016 / Accepted: 6 June 2017 / Published online: 15 July 2017

(C) The Author(s) 2017. This article is an open access publication

\begin{abstract}
This paper focuses on estimating real and quantum potentials from financial commodities. The log returns of six common commodities are considered. We find that some phenomena, such as the vertical potential walls and the time scale issue of the variation on returns, also exists in commodity markets. By comparing the quantum and classical potentials, we attempt to demonstrate that the information within these two types of potentials is different. We believe this empirical result is consistent with the theoretical assumption that quantum potentials (when embedded into social science contexts) may contain some social cognitive or market psychological information, while classical potentials mainly reflect 'hard' market conditions. We also compare the two potential forces and explore their relationship by simply estimating the Pearson correlation between them. The Medium or weak interaction effect may indicate that the cognitive system among traders may be affected by those 'hard' market conditions.
\end{abstract}

Keywords Quantum potential · Commodity markets · Information

\section{Introduction}

The area of academic finance is not foreign to using concepts from other disciplines to explain financial phenomena. The area of applied physics has provided for a possible toolkit to explain and model some financial phenomena. For example, in their book, Mantegna and Stanley [1] conclude that by utilizing statistical and probabilistic approaches (taken from statistical physics) in financial time series, they get new ideas about the behaviour of financial markets. Another example is chaos theory where physics methods turn out to be very useful in the analysis of complex economic systems (Chakraborti [2]). See also Chen [3].

E. Haven

eh76@le.ac.uk

1 School of Business, University of Leicester, Leicester, UK 
Khrennikov [4] was the first to introduce formalisms from quantum mechanics to a set of fields of application outside of quantum mechanics, such as psychology; finance; economics and also biology. See Khrennikov [5-7]. A very intense movement of research has now come to fruition where investigations show that elements of the quantum formalism can be used to great benefit in the modelling of decision making and other areas. We note, amongst others, the work by Busemeyer [8]; Aerts, Sozzo and Veloz [9] and Brandenburger [10].

We may wonder whether it is palatable to claim that a lot of the research which uses quantum formalisms in social science, is concerned with the provision of a new model. As an example, the use of quantum probability in decision making can be argued to serve as an input for new models in decision making. On the contrary, it is not unreasonable to argue that there is less 'model-building' going on when one considers the application of formalisms from statistical mechanics to finance and economics. An exception is surely the work by Baaquie (see $[11,12]$ ), which very much explicitly builds up basic concepts from economics from the perspective of statistical mechanics, i.e. with the use of real potential functions and Hamiltonian dynamics.

Our paper has as objective to consider an application of the quantum formalism to finance. So far, such applications have been fairly sparse. We can mention the contributions of Fisher information to asset pricing with the work of Hawkins and Frieden [13]. Recently, Tahmasebi et al. [14] have applied the concept of quantum potential in finance from an empirical perspective. Our paper uses pilot wave theory to model quantum potentials from financial commodities. The key works which provide for the foundations of pilot wave theory are: Bohm [15, 16]; Bohm and Hiley [17]. See also Holland [18]. There have been applications of pilot wave theory to finance. See Khrennikov [5, 6]; Choustova [19]; Haven and Khrennikov [20] and Haven [21]. An important element in this approach to finance is that borrowing from pilot-wave theory allows for a formalism, which we could call, information dynamics.

Recent work using empirical evidence in the application of some elements of the pilot wave formalism to finance is by Tahmasebi et al. [14]. The authors analyze the time series of the S\&P500 index. In this paper, we will follow the logic of Tahmasebi et al. [14] to calculate the quantum potential. However, we will focus on data from commodity markets rather than on data from security markets. The return time series of six commodities which are 'Brent oil', 'heat oil', 'gold', 'silver', 'copper' and 'corn' are selected from 'Bloomberg Professional'. For different aims of comparisons, different time intervals of data are utilized. For example, we use the daily returns of gold over 36 years from the 1st of January 1980 to the 30th of June 2016. but a much shorter time interval (only 500 recent trading days ending on 31th May 2016) is chosen for other discussions. In our analysis we also consider the real potential and the interpretation of potential force is also considered.

In the next section we provide for an overview of the essential formalism which renders the quantum potential. We then pursue the same goal to discuss how we might obtain the real potential within the context of the Fokker-Planck PDE. In Section 4, we consider the kernel density estimation method and in Section 5, we discuss the empirical results. We conclude in Section 6.

\section{The Mathematical Formalism of the Quantum Potential}

In this section, we provide for some of the essential steps which yield the so called quantum potential. We follow here Haven and Khrennikov [20]. See also Holland [18] for more detail. 
The polar form of the wave function ${ }^{1} \psi(q, t)=R(q, t) \exp \left(i \frac{S(q, t)}{h}\right)$, is inserted into the Schrödinger PDE:

$$
i h \frac{\partial \psi}{\partial t}=-\frac{h^{2}}{2 m} \frac{\partial^{2} \psi}{\partial q^{2}}+V(q, t) \psi(q, t) ;
$$

where $V(q, t)$ is the time dependent real potential; $q$ is position; $h$ is the Planck constant; and $m$ is mass.

After some steps, one obtains two central equations:

$$
\frac{\partial R^{2}}{\partial t}+\frac{1}{m} \frac{\partial}{\partial q}\left(R^{2} \frac{\partial S}{\partial q}\right)=0
$$

and also:

$$
\frac{\partial S}{\partial t}+\frac{1}{2 m}\left(\frac{\partial S}{\partial q}\right)^{2}+\left(V-\frac{h^{2}}{2 m R} \frac{\partial^{2} R}{\partial q^{2}}\right)=0 .
$$

Equation (2) is known as the so called continuity equation. It indicates the time evolution of the probability density function $R^{2}=|\psi|^{2}$. For our purposes, Equation (3) is important and Bohm [15, 16]; Bohm and Hiley [17] will interpret $\frac{h^{2}}{2 m R} \frac{\partial^{2} R}{\partial q^{2}}$ as the so called quantum potential.

A Newton-Bohm equation can be obtained:

$$
m \frac{d^{2} q(t)}{d t^{2}}=-\frac{\partial U(q, t)}{\partial q}-\frac{\partial V(q, t)}{\partial q} ;
$$

where $U(q, t)=\frac{h^{2}}{2 m R} \frac{\partial^{2} R}{\partial q^{2}}$.

The dynamics given by the Newton-Bohm equation cannot be considered independent of the Schrödinger's dynamics.

\section{The Mathematical Formalism of the Classical Quantum Potential}

For completeness, we simply state the mathematical formalism of the classical (real) potential within the context of the Fokker-Planck PDE. We follow Haken [22]. Starting from the so-called Fokker-Planck PDE, which describes the time evolution of the probability density function of the position of a particle:

$$
\frac{\partial f(q, t)}{\partial t}=\frac{\partial}{\partial q} \gamma q f(q, t)+\frac{1}{2} Q \frac{\partial^{2}}{\partial q^{2}} f(q, t) ;
$$

where $K=-\gamma q$ is the drift coefficient whilst $Q$ is called the diffusion coefficient and $f(q, t)$ is the probability density function. The above one-dimensional Fokker-Planck PDE can be rewritten as a continuity equation in the form:

$$
\frac{\partial f(q, t)}{\partial t}+\frac{\partial}{\partial q}\left(K f(q, t)-\frac{1}{2} Q \frac{\partial f(q, t)}{\partial q}\right)=0 .
$$

If the probability density function is time-independent, then we obtain the equation:

$$
j=\left(K f(q)-\frac{1}{2} Q \frac{\partial f(q)}{\partial q}\right)=\text { const } .
$$

\footnotetext{
${ }^{1} R$ is the amplitude and $S$ is the phase.
} 
According to the natural boundary condition on the probability density function $f, f$ vanishes for $q \rightarrow \pm \infty$ which implies that $j \rightarrow 0$ for $q \rightarrow \pm \infty$. See Haken [22]. As a result, the constant in the above equation must vanish. So one obtains:

$$
\frac{1}{2} Q \frac{\partial f(q)}{\partial q}=K f(q)
$$

The above ordinary differential equation can be solved by:

$$
f(q)=N \exp \left(-\frac{2 V(q)}{Q}\right) ;
$$

where the classical potential is denoted as:

$$
V(q)=-\int K(q) d q
$$

and the constant $N$ is determined by:

$$
\int_{-\infty}^{+\infty} f(q) d q=1 .
$$

\section{The Kernel Density Estimation Method}

In this paper, we adopt the so called Kernel density estimation (KDE) method to extract the probability density function from the empirical data that we selected. In statistics, Kernel density estimation was introduced by Rosenblatt [23] and it is a non-parametric way to estimate the density of a random variable. One of the advantages in using the KDE method is that it has a relatively simple form and is easy to implement. If each state $q_{i}$ of a return time series is independent and identically distributed, then the kernel density estimator can be written as:

$$
f_{b}(x)=\frac{1}{n b} \sum_{i=1}^{n} K\left(\frac{q-q_{i}}{b}\right) ;
$$

where $K$ is a non-negative kernel function that integrates to one and has mean zero; $b$ is a smoothing parameter with positive value and is called the bandwidth; $n$ is the size of the random variable. There are a range of different kernel functions which are commonly used. In this paper, the popular Gaussian kernel function, namely:

$$
K(y)=\frac{1}{\sqrt{2 \pi}} \exp \left(-\frac{y^{2}}{2}\right)
$$

is selected. We need also to find an appropriate bandwidth $b$ for each time series. According to the definition of the asymptotic mean integrated squared error, the best bandwidth is given by:

$$
b=\left(\frac{R(K)}{\mu_{2}(K)^{2} R\left(f^{\prime \prime}\right)}\right)^{\frac{1}{5}} n^{-\frac{1}{5}} ;
$$

where $R(K)=\int K^{2}(y) d y$ and $R\left(f^{\prime \prime}\right)=\int\left(f^{\prime \prime}(y)\right)^{2} d y$ (see Sheather [24]). However, the term $f^{\prime \prime}(y)$ is unknown in the above equation. Often, an approximation to the above equation is used. In this paper, we follow Silverman's [25] rule of thumb method and reduce the above equation to:

$$
b=1.06 \sigma n^{-\frac{1}{5}}
$$




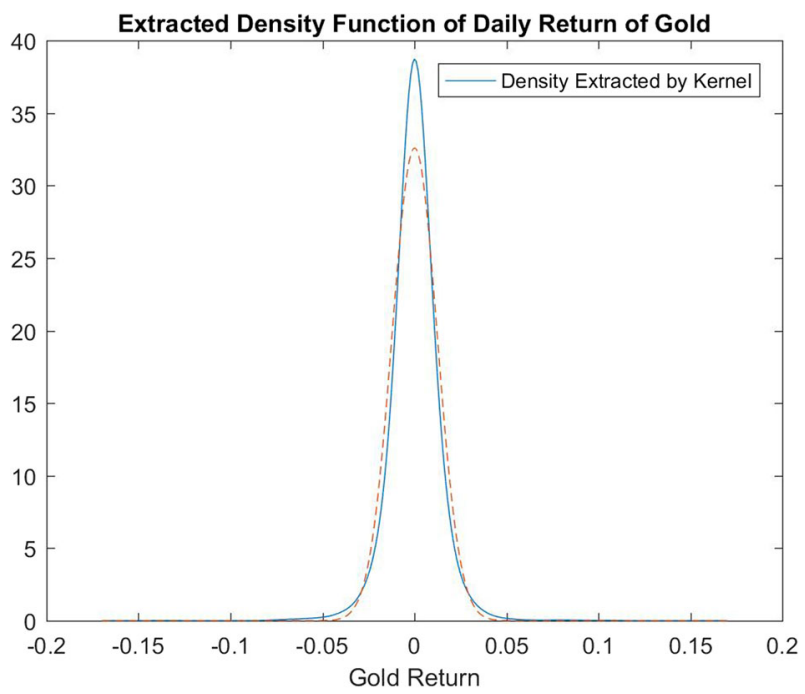

Fig. 1 The extracted density function figure of daily gold return. The bandwidth selected in $1 \mathrm{~b}$ is 0.006 and the dashed curve is a Gaussian function with the same mean value as well as the variance

where $\sigma$ is the sample standard deviation. It has to be pointed out that the bandwidth $b$ is related to the smoothness of the estimated density function. The larger $b$ is, the smoother the density function will be. The use of an approximation of bandwidth may influence the precision of our extracted density function. But since this paper focuses more on the trend of curves rather than the exact values, such inaccuracy can be tolerated. This will be also mentioned when the comparisons are implemented in the following section.

\section{The Empirical Results}

The extracted density function for daily returns of gold $^{2}$ by applying the Kernel density estimation (KDE) method is shown in Fig. 1. One intuitive phenomenon from Fig. 1 is that the extracted density distribution by the KDE method is a fat-tailed distribution and it exhibits relatively large kurtosis, which is consistent with a lot of finance research on the time series in commodity markets.

The quantum potentials of return time series of gold in daily, monthly and yearly scales are illustrated in respectively Figs. 2, 3 and 4 with $h$ and $m$ being equal to 1 .

We observe that for all three types of data with different time scales, there are a pair of potential walls which prevent the returns from deviating too far away from the mean values. By comparing Figs. 2, 3 and 4 different change tolerances are illustrated. For the daily time scale, returns can change within the range from the value around -0.15 to 0.13 . For monthly data, the variation is from -0.27 to 0.24 . Finally, the yearly time scale allows the largest variation which is from -0.5 to 0.4 . Those results are similar to what Tahmasebi et al. [14] find when they plot the quantum potential for the security market (S\&P500 index). They point out that the quantum potential causes restrictions for amplitude changes with respect to time scales of data.

\footnotetext{
${ }^{2}$ As mentioned before, all the data selected in this paper comes from Bloomberg Professional.
} 


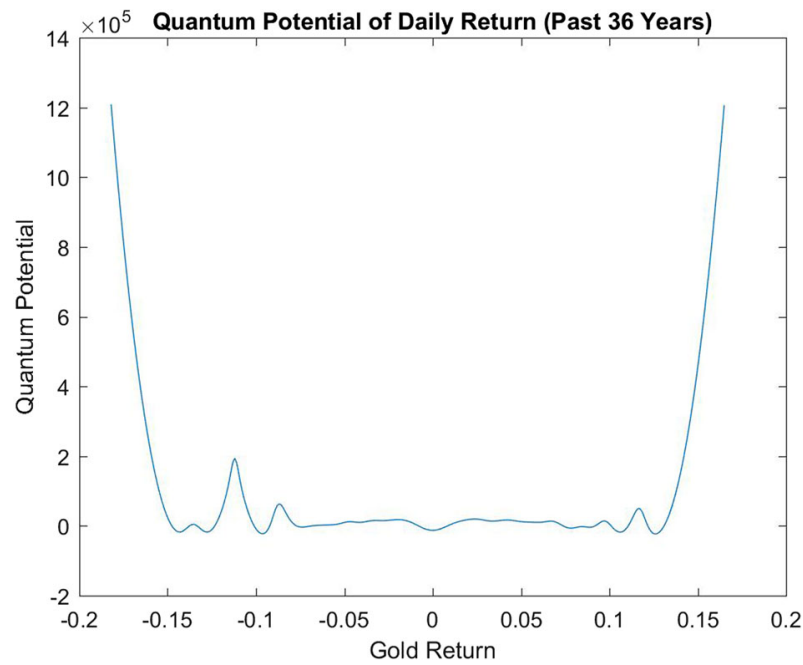

Fig. 2 Quantum potential for gold - daily return

We consider other commodities in the below Figs. 5, 6, 7, 8, 9 and 10 (all daily returns). The dotted curve in Figs. 5-10, is the quantum potential calculated from the Gaussian density function $f(x)=\frac{1}{\sqrt{2 \pi} \sigma} \exp \left(-\frac{\left(q-q_{0}\right)^{2}}{2 \sigma^{2}}\right)$. When using this function in the quantum potential $U(q, t)=\frac{h^{2}}{2 m R} \frac{\partial^{2} R}{\partial q^{2}}$, we get:

$$
U=\frac{\left(q-q_{0}\right)^{2}}{\sigma^{4}}-\frac{2}{\sigma^{2}}
$$

where $q_{0}$ is the mean value and $\sigma^{2}$ is the variance.

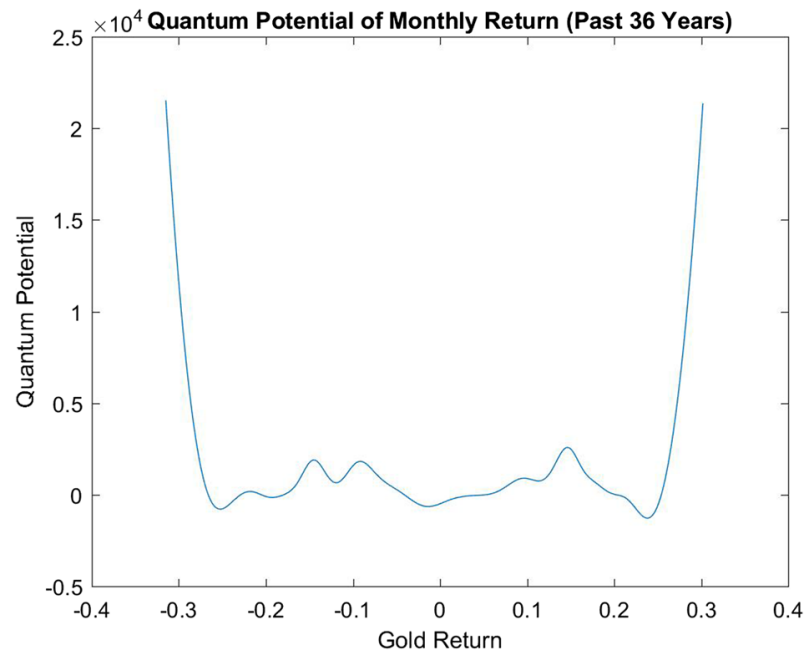

Fig. 3 Quantum potential for gold - Monthly return 


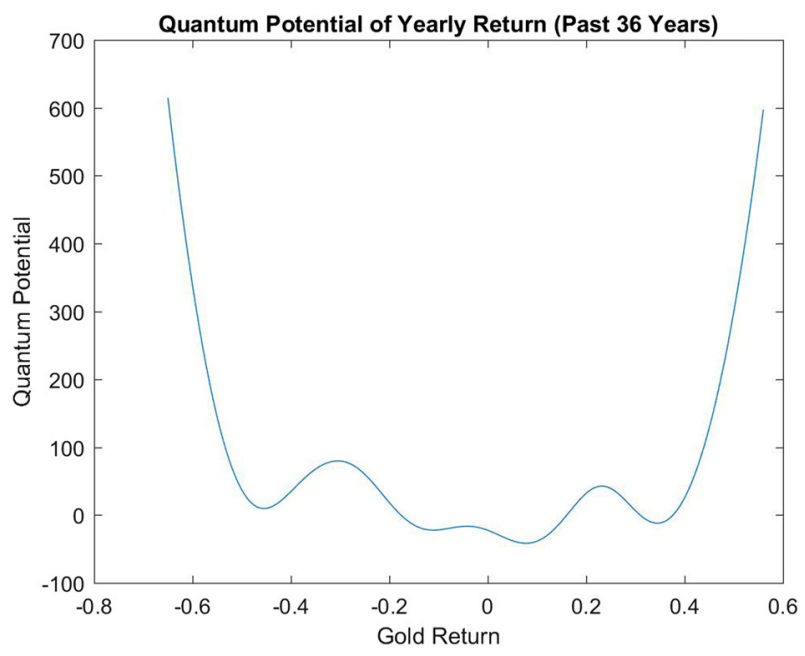

Fig. 4 Quantum potential for gold - Yearly return

The Figs. 5-10, below combine the classical and quantum potentials for various commodities. It can be observed that if the probability density function of returns is a Gaussian function, then there are no potential walls and some values, which are far removed from the mean value are possible. Another interesting phenomenon is that potential values of extracted and Gaussian densities are generally overlapping better in the central part around the mean value (as opposed to the two extreme sides near the potential walls). If we consider the common fat tail phenomenon of the distribution of returns, it seem to suggest that the quantum potential may contain some kind of information which could be used to explain a relatively higher probability and higher frequency to obtain larger returns than is the case with the normal distribution for real financial data.

In the same Figs. 5-10, we can also observe the curves of the classical (real) potential derived from (9) with diffusion coefficient $Q$ equal to 1 . Since the parameters $h$ and $m$ in

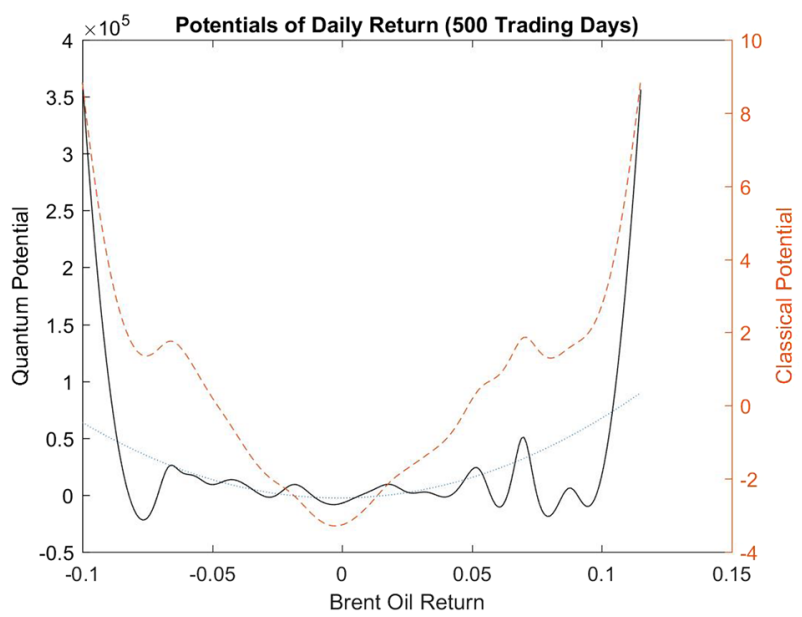

Fig. $5 U, V$ for Brent Oil. Note the $U$ of a Gaussian density function (dotted curve) and the classical potential (dashed curve) with the diffusion coefficient $\mathrm{Q}$ is equal to 1 


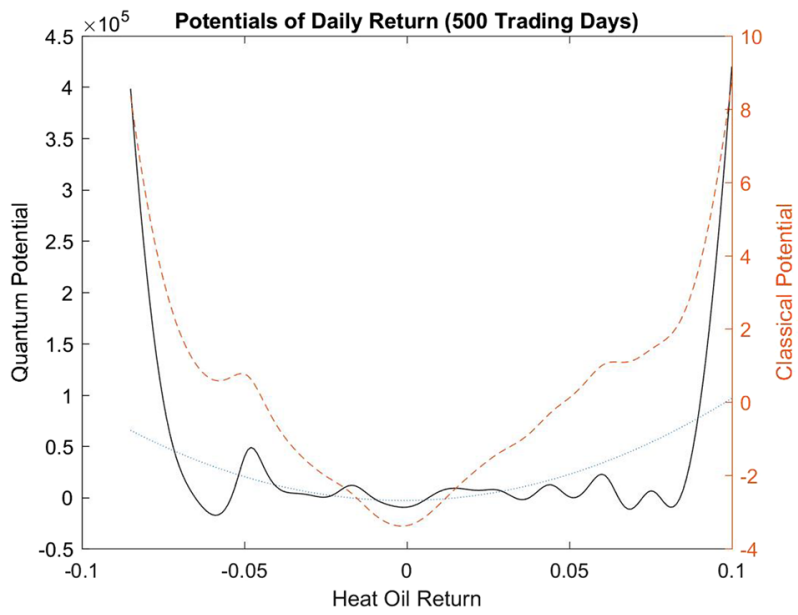

Fig. $6 U, V$ for Heating Oil. Note the $U$ of a Gaussian density function (dotted curve) and the classical potential (dashed curve) with the diffusion coefficient $\mathrm{Q}$ is equal to 1

the quantum potential $U$ are not estimated, the values of these two types of potentials cannot be compared directly due to the scaling issue. However, a comparison of the shapes can still be implemented.

On the one hand, the classical potential is very close to an inverted bell shape curve with two tails increasing to infinite. The minimum of the potential is at the mean value of returns, so there is an equilibrium point at the mean value where the force related to this type of potential is equal to 0 . On the other hand, there is a pair of potential walls for the quantum potential whilst between the walls, the curves are relatively plain and do not have a gradually increasing trend when the distance from the mean value becomes larger, which is quite unlike the classical potential. The different shapes of the two types of potentials may indicate that the information included in them is of a different type. In fact, as has

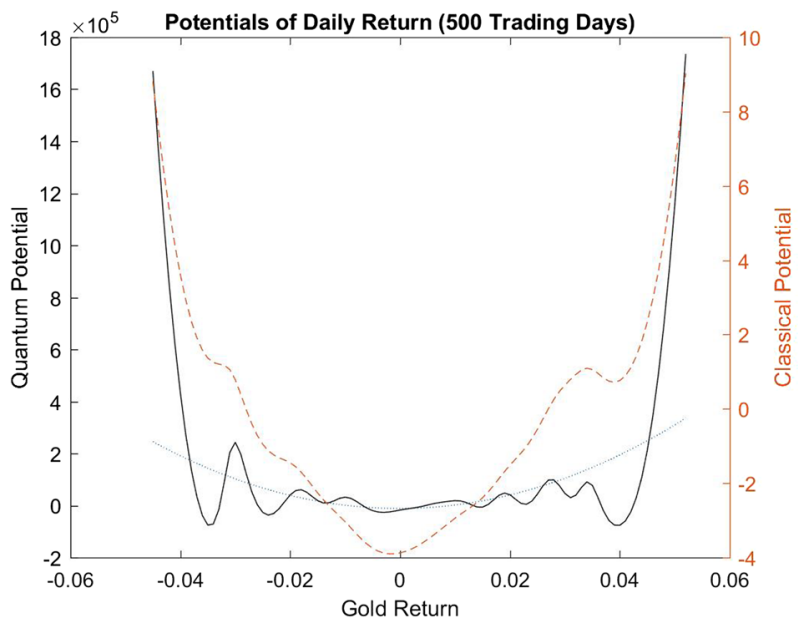

Fig. $7 U, V$ for Gold. Note the $U$ of a Gaussian density function (dotted curve) and the classical potential (dashed curve) with the diffusion coefficient $\mathrm{Q}$ is equal to 1 


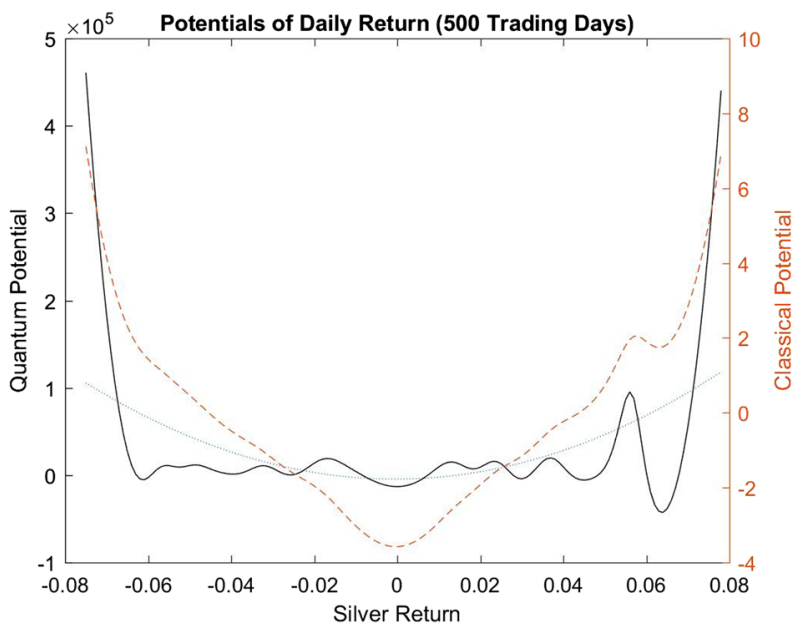

Fig. $8 U, V$ for Silver. Note the $U$ of a Gaussian density function (dotted curve) and the classical potential (dashed curve) with the diffusion coefficient $\mathrm{Q}$ is equal to 1

been remarked by Khrennikov [6] (see also Haven and Khrennikov [20]) that apart from the traditional 'hard' financial conditions (levels of input for instance), there exists information reflecting social cognitive and psychological factors and those could be described by an information 'field' $\psi(q, t)$ which can also affect the price and price-change (return). The quantum potential derived from the pilot wave $\psi(q, t)$ as shown in the figures seems to suggest that within the range of possible returns, some large values may contain similar psychological or mental information.

In the figures above, there are some peaks appearing in the quantum potential curves. For example, in Fig. 7, a significant peak is presented for the value of gold return being smaller than -0.03 . For the real potential, a point near the 0.06 return in Fig. 8, shows an equilibrium value other than the mean value. How could this be caused? Firstly, this may be

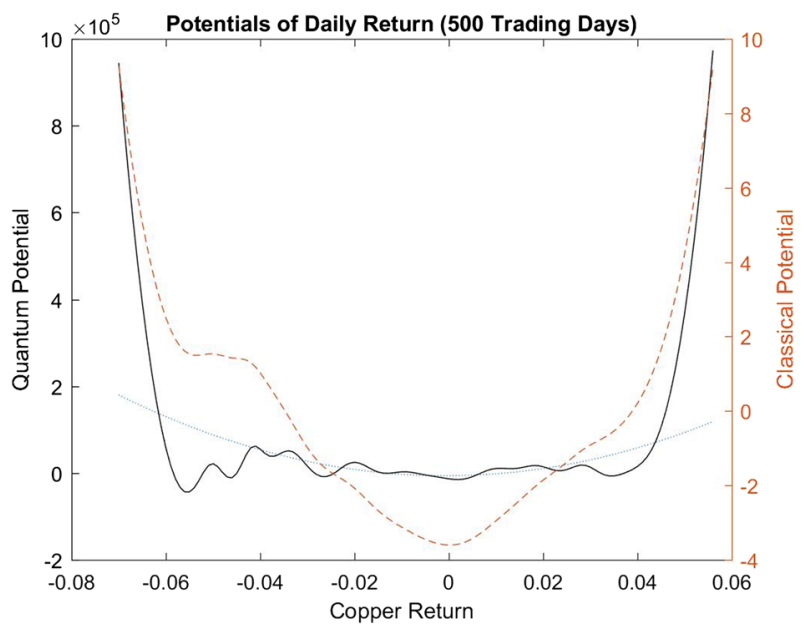

Fig. $9 U, V$ for Copper Return. Note the $U$ of a Gaussian density function (dotted curve) and the classical potential (dashed curve) with the diffusion coefficient $\mathrm{Q}$ is equal to 1 


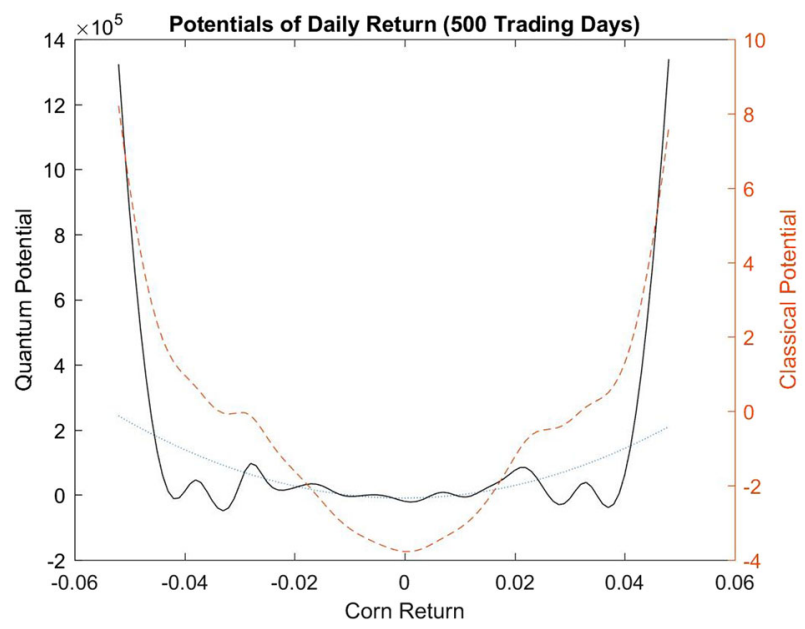

Fig. $10 U, V$ for Corn. Note the $U$ of a Gaussian density function (dotted curve) and the classical potential (dashed curve) with the diffusion coefficient $\mathrm{Q}$ is equal to 1

caused by the KDE method which is adopted to estimate the probability density function. Since the real empirical data is irregular and discontinuous in some interval, the smoothness of the estimated function will much depend on the selected bandwidth. If we increase the bandwidth, the probability density function will be more smooth and the peaks as well as the unexpected equilibria may disappear. However, we know that obtaining the 'best' bandwidth is a relatively complex statistical problem and in order to simplify the calculation process, an approximation under the criterion of (15) is utilized in this paper. As a consequence, it is probable one can obtain under-smoothed curves. Moreover, the precision of smoothness does not affect the integral shapes of the two types of potentials, so the comparisons between them are not affected by this issue.

We can also find the force. Two types of financial forces can be found (i.e. on the basis of the real and quantum potentials). Figure 11 gives an example of these financial forces with the data input of daily return time series of corn.

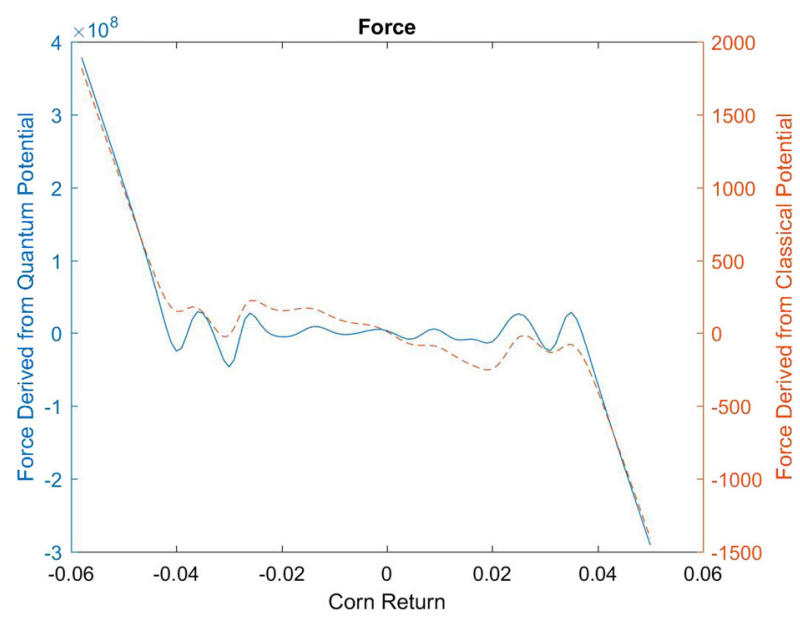

Fig. 11 The forces (gradients) derived from the quantum and classical (dashed curve) potentials 
Table 1 The Pearson correlation coefficient of two forces within the range of possible returns

\begin{tabular}{llll}
\hline & $\begin{array}{l}\text { Range of possible } \\
\text { return }\end{array}$ & $\begin{array}{l}\text { Pearson correlation } \\
\text { coefficient }\end{array}$ & p-value \\
\hline Brent oil & {$[-0.0777,0.0927]$} & 0.4570 & 0.0000 \\
Heat & {$[-0.0653,0.0796]$} & 0.4206 & 0.0000 \\
Gold & {$[-0.0338,0.0406]$} & 0.3213 & 0.0049 \\
Silver & {$[-0.0599,0.0632]$} & 0.3877 & 0.0000 \\
Copper & {$[-0.0547,0.0407]$} & 0.2737 & 0.0070 \\
Corn & {$[-0.0405,0.0366]$} & 0.2655 & 0.0188 \\
\hline
\end{tabular}

Due to the scaling issue about the un-evaluated $h$ and $m$ and the diffusion coefficient $Q$, the comparison of values is omitted in this paper. We focus on the shapes instead. Figure 11 shows that the shapes of these two types of forces are quite similar. Both derived forces restrict the variation of returns in a range. Specifically, if the returns try to jump well out of range, a strong negative reaction force will pull those returns back. Moreover, within the range, the financial force based on the classical potential also has a negative reaction trend which prevents returns from deviating from the mean value. However, the slope of such negative reaction trend is much smaller within the range as opposed to the trend when out of range values are considered. As an example, for the force derived from the classical potential, we see strong forces, for out of range values outside of the interval $[-0.04,0.04]$. We observe a much weaker slope for values within the interval [ $-0.04,0.04]$.

For the financial force derived from the quantum potential, we can observe that for inrange values, i.e. within again the interval $[-0.04,0.04]$, there is a weak negative reaction trend (weaker than in the case of the force derived from the classical potential). Out of range values are again characterized by strong negative reaction forces. Those differences in forces derived from either the real or quantum potential may possibly account for empirical evidence illustrating the difference between so-called 'hard' and 'soft' financial factors.

The table above shows that the gradients of the two potentials do have positive correlations, but the level of such correlation is not very strong (Table 1).

The fairly weak correlation of in-range values between the two types of forces does need some explanation. For the forces derived from the real potential, the argument that undervalued assets are bought and overvalued assets are sold seem to indicate there is an inverse force which needs to keep prices steady. This steadiness of prices may be the result of an efficient market hypothesis. But there is more to price behavior than just steadiness of prices. The force derived from the quantum potential which contains social, cognitive and psychological information may also exist and have an effect on traders. And the force for within range values is less pronounced than it is for the case of the force derived from the classical potential. As a result, one might claim that traders with different expectations can tolerate large price changes and will not take action to invert them, and this may explain the speculative behaviour in financial commodity markets for instance.

\section{Conclusion}

In this paper we have derived the quantum and real potentials and their respective forces for returns on a set of six financial commodities. We have pointed out that by applying the KDE method, the extracted probability density function is sensitive to the parameter bandwidth 
and the selected bandwidth under the criterion adopted may not be the best one. However, the relatively imprecise bandwidth does not affect shapes and trends of the curves that we plotted and the following conclusions can be obtained.

For the commodity markets data, there exists two potential walls which are the boundaries of the fluctuations of possible return values and the range of variation depends on the time scales. In detail, yearly data has the largest variation range whilst daily data has the smallest one. These phenomena are confirming the results of Tahmasebi et al. [14] who mainly focus on security markets. Then we compare the quantum potential of the extracted density from empirical data with the Gaussian density. The quantum potential of the Gaussian density does not have potential walls. Hence, larger or smaller values may be reached with low probability, and this is also consistent with the finding of Tahmasebi et al. [14].

We also compare the quantum potential with the classical potential. Due to scaling issues, the comparisons just focus on the shapes of curves. The significant different shapes of the two types of potential, demonstrate that they may contain different kinds of information. We discussed the 'hard' financial factors versus the social cognitive and psychological factors described by the pilot wave.

The flatter slope of the force derived from the quantum potential for in-range values, may indicate that from the point of view of social cognitive or psychological aspects, traders may not implement the inverse trading strategies when price changes and their speculative behavior may easily lead to relatively large or small returns. Finally, the positive but small correlation coefficients between the two types of forces implies that these two forces may have some medium or weak interaction effect. This can be easily understood since the cognitive system among traders may be affected by economic and financial conditions, but may not be determined by it.

Open Access This article is distributed under the terms of the Creative Commons Attribution 4.0 International License (http://creativecommons.org/licenses/by/4.0/), which permits unrestricted use, distribution, and reproduction in any medium, provided you give appropriate credit to the original author(s) and the source, provide a link to the Creative Commons license, and indicate if changes were made.

\section{References}

1. Mantegna, R.N., Stanley, H.E.: An introduction to econophysics: Correlation and complexity in finance. Cambridge University Press, Cambridge (2000)

2. Chakraborti, A., Toke, I., Patriarca, M., Abergel, F.: Econophysics: Empirical facts and agent-based models. ArXiv: Cornell University: arXiv.org > q-fin > arXiv:0909.1974 (2009)

3. Chen, J.: The unity of science and economics: a new foundation of economic theory. Springer, New York (2015)

4. Khrennikov, A.: Classical and quantum mechanics on information spaces with applications to cognitive, psychological, social and anomalous phenomena. Found. Phys. 29, 1065-1068 (1999)

5. Khrennikov, A.: Information dynamics in cognitive, psychological, and anomalous phenomena. Series Fundamental Theories of Physics, vol. 138. Kluwer, Dordrecht (2004)

6. Khrennikov, A.: Ubiquitous quantum structure. Springer, Heidelberg (2010)

7. Asano, M., Khrennikov, A., Ohya, M., Tanaka, Y., Yamato, I.: Quantum adaptivity in biology: from genetics to cognition. Springer, Heidelberg-Berlin-New York (2015)

8. Busemeyer, J.R., Wang, Z., Townsend, J.T.: Quantum dynamics of human decision making. J. Math. Psychol. 50, 220-241 (2006)

9. Aerts, D., Sozzo, S., Veloz, T.: The quantum nature of identity in human thought: Bose-Einstein statistics for conceptual indistinguishability. Int. J. Theor. Phys. 54, 4430-4443 (2015)

10. Brandenburger, A.: The relationship between quantum and classical correlation in games. Game Econ. Behav. 69, 175-183 (2010)

11. Baaquie, B.: Statistical Microeconomics. Physica A 392(19), 4400-4416 (2013) 
12. Baaquie, B., Du, X., Bhanap, J.: Option pricing: Stock price, stock velocity and the acceleration Lagrangian. Physica A 416, 564-581 (2014)

13. Hawkins, R.J., Frieden, B.R.: Asymmetric information and quantization in financial economics. In: International Journal of Mathematics and Mathematical Sciences, pp. 1-11 (2012)

14. Tahmasebi, F., Meskinimood, S., Namaki, A., Farahani, S.V., Jalalzadeh, S., Jafari, G.R.: Financial market images: a practical approach owing to the secret quantum potential. Europhys. Lett. 109(3), 30001 (2015)

15. Bohm, D.: A suggested interpretation of the quantum theory in terms of hidden variables. Phys. Rev. 85, 166-179 (1952a)

16. Bohm, D.: A suggested interpretation of the quantum theory in terms of hidden variables. Phys. Rev. 85, 180-193 (1952b)

17. Bohm, D., Hiley, B.: The undivided universe: an ontological interpretation of quantum mechanics. Routledge and Kegan Paul, London (1993)

18. Holland, P.: The quantum theory of motion: an account of the de broglie-bohm causal interpretation of quantum mechanics. University Press, Cambridge (2000)

19. Choustova, O.: Quantum model for the price dynamics: the problem of smoothness of trajectories. J. Math. Anal. Appl. 346, 296-304 (2008)

20. Haven, E., Khrennikov, A.: Quantum social science. University Press, Cambridge (2013)

21. Haven, E.: Pilot-wave theory and financial option pricing. Int. J. Theor. Phys. 44(11), 1957-1962 (2006)

22. Haken, H.: Synergetics: An Introduction: Nonequilibrium Phase Transitions and Self-Organization in Physics, Chemistry and Biology. Springer, Heidelberg (1983)

23. Rosenblatt, M.: Remarks on some nonparametric estimates of a density function. Ann. Math. Stat. 27(3), 832-837 (1956)

24. Sheather, S.J.: Density estimation. Stat. Sci. 19(4), 588-597 (2004)

25. Silverman, B.W.: Density estimation for statistics and data analysis. Chapman and Hall, London (1986) 